\title{
Ankle arthroscopy
}

\begin{abstract}
Ankle pain is a common musculoskeletal problem, affecting more than $10 \%$ of the total Greek population. In a case series examining arthroscopic management of anterior ankle impingement (AAI), ${ }^{1} 90 \%$ of patients with AAI were found to have moderate to severe limitation in activities of daily living due to pain. ${ }^{2} \mathrm{AAI}$ is defined by anterior ankle pain and painful terminal dorsiflexion on physical examination. Weight-bearing radiographs typically show so-called kissing exostoses on the anterior distal tibia and dorsal talus. The tibiotalar joint space is preserved; this feature distinguishes AAI from early ankle osteoarthrosis. Both nonsurgical and surgical management have proved to be successful. Surgically, AAI has traditionally been treated with open débridement. Arthroscopic minimally invasive techniques have become more popular in recent decades, ${ }^{3}$ however. In 1949, McMurray T ${ }^{4}$ penned his final publication, titled "Footballer's Ankle." In this classic article, McMurray described a chronic ankle injury found in professional soccer players who reported point tenderness over the anterior ankle joint and pain with passive dorsiflexion. ${ }^{5}$ Radiographs showed ankle osteophytes. ${ }^{6}$ Intraoperatively, exostoses were found deep to the joint capsule in the setting of a healthy articular surface. This finding was in stark contrast to the eburnated articular surface seen in osteoarthritis. McMurray $\mathrm{T}^{4}$ treated six professional footballers with open decompression, all of whom were able to return to professional athletics. The 1980s saw renewed interest in AAI. Since then, research has focused on the etiology and management of AAI.
\end{abstract}

Keywords: Ankle Arthroscopy, Anterior ankle impingement, Ligaments, Ankle pain
Volume 3 Issue 3 - 2015

\section{Evangelos Evangelou \\ Orthopaedic Clinic, Metropolitan Hospital Athens, Greece}

Correspondence: Evangelos Evangelou, Orthopaedic Clinic, Metropolitan Hospital Athens 90, Heroes Politechniou, Piraeus, Athens, Greece, Tel 0030 6977716739,

Email footanklesurgery.gr, vagevangelou@gmail.com

Received: September 03, 2014 | Published: October 06, 2015

\section{Anatomy}

The ankle is an imperfect and highly constrained hinge joint composed of three articulations: the tibiotalar, fibulotalar, and tibiofibular joints. ${ }^{7}$ The distal tibia and fibula form the ankle mortise, with the trochlea of the talus acting as the keystone. This trochlea has been likened to a truncated cone with its apex oriented medially. From above, the talus appears wedge-shaped, narrowing posteriorly. This shape contributes to ankle containment and stability. Motion at the ankle is multiplanar, with an obliquely oriented axis of rotation that runs through the tips of the malleoli. This axis of rotation results in downward and posterior inclination in the sagittal plane and posteromedial inclination in the transverse plane. The end result is a combined rolling movement of flexion-extension associated with a horizontal sliding rotation and coronal plane abduction adduction. The three articulations of the ankle are stabilized by a balance of static and dynamic structures. The ligaments that impart stability are divided into three groups: syndesmotic, lateral, and medial. At the level of the tibial plafond lie the syndesmosis and its encompassing ligaments, which maintain the relationship of the distal fibula and tibia. ${ }^{8}$ These are the anterior inferior tibiofibular ligament (AITFL), posteriorinferior tibiofibular ligament, transverse tibiofibular ligament, ${ }^{9}$ and interosseous tibiofibular ligament. This strong ligament complex, in combination with the bony architecture, prevents proximal migration of the talus between the tibia and fibula and contributes transverse stability to motion of the tibiofibular joint. The lateral ligaments consist of the anterior talofibular ligament, the calcaneofibular ligament, and the posterior talofibular ligament. This ligamentous complex is the primary restraint to anterior translation of the talus. The medial ligaments are the superficial deltoid, deep deltoid, and spring (i.e., plantar calcaneonavicular). These ligaments resist posterior and lateral translation as well as valgus tilt of the talus. ${ }^{10}$ Together, these ligaments play a crucial role in guiding stable joint motion. Damage to these structures will affect the coupled motion of the intact ankle joint. Further, ligamentous instability may coexist and play a role in the etiology of AAI. Thus, a thorough understanding of the original geometry of the ankle complex is crucial to restoration of function.

\section{Pathophysiology}

The exact cause of AAI remains unclear. McMurray proposed a "pull" etiology on the bone spurs and thought that AAI initiated with traction injuries to the anterior capsule. ${ }^{11}$ O'Donoghue ${ }^{12}$ disagreed, believing repetitive impact from forced dorsiflexion to be the cause of bone spur formation. McMurray's hypothesis of a traction etiology leading to exostosis formation is unlikely because the exostoses are found deep to the joint capsule rather than within the substance of the capsule. Van Dijk, for example, noted that the exostoses on the tibia and talus tend to be intra-articular and not attached to any capsular structures that would impart traction. Activities that produce repetitive trauma seem to be more correlated to the development of AAI. ${ }^{13}$ Dance is another activity in which the ankle may be subjected to repetitive abnormal motions.

The plié, consisting of forced dorsiflexion with locked external rotation, has been described as the most common movement contributing to AAI. Soft-tissue structures can cause AAI without actual bony impingement, ${ }^{14}$ however Berberian et al ${ }^{15}$ performed CT scans on 9 patients (10 ankles) with AAI and found that the talar spur lies medial to the midline, whereas the tibial spur lies lateral to the midline; typically, the spurs do not overlap. A triangular shaped area of soft tissue consisting of synovium, collagen, blood vessels, and adipose is located in the anterior joint space between the talus and tibia; in a normal ankle, these tissues are compressed between the talus and tibia on dorsiflexion.

In patients with AAI syndrome, pain may be secondary to impingement of this soft tissue ${ }^{16}$ Coincident chondral and osteochondral lesions may be found in patients with anterior ankle pain. ${ }^{17}$ Rasmussen et al performed 105 ankle arthroscopies in patients with painful dorsiflexion and found 20 chondral lesions and 16 loose bodies. In a series of ankle arthroscopies for exostoses resection, 
Moon et al found that $80.7 \%$ of patients with distal tibial exostoses also had a corresponding cartilage lesion on the talar dome. ${ }^{18}$ Ankle instability may also contribute to AAI syndrome. Between $13 \%$ and $35 \%$ of patients continue to have ankle pain after a lateral ankle stabilization procedure. This may be due to unaddressed intraarticular pathology.

\section{Patient evaluation}

\section{Physical examination}

Despite controversy regarding the underlying pathophysiology of AAI, its clinical presentation is consistent and straightforward. In the early stages of the disorder, anterior ankle pain is elicited with long periods of exercise and is relieved with rest. ${ }^{19}$ As AAI becomes chronic, additional symptoms may include instability; limited ankle motion; and pain with squatting, sprinting, stair climbing, and hill climbing. Normal gait may be unaffected. Physical examination typically elicits tenderness over the anterior ankle joint and pain with forced dorsiflexion. Ankle swelling maybe present, as well. Patients also should be evaluated for other etiologies or concurrent ankle pathology. The physical examination should include inspection of the ankle for swelling, erythema, and alignment. An anterior drawer test should be performed to evaluate for lateral ankle instability. ${ }^{20}$ The Silfverskiöld test should be performed to evaluate for isolated gastrocnemius contracture. This test measures ankle dorsiflexion with the knee in extension and in $90^{\circ}$ of flexion. The test is considered positive when ankle dorsiflexion is greater with the knee in flexion than in extension.

\section{Imaging}

The diagnosis of AAI is usually made clinically and confirmed with plain radiographs. Patients often have exostoses on the distal anterior tibia and dorsal talar neck. Unlike the presentation in ankle osteoarthritis, the joint space is preserved on weight-bearing lateral ankle radiographs. ${ }^{21}$ Anteromedial exostoses may not be visible on lateral radiographs due to the presence of superimposed structures. CT detects the presence of exostoses, and MRI is useful in evaluating for soft-tissue impingement as well as chondral or subchondral injuries. ${ }^{22}$ Huh et al showed that MRI has $92 \%$ sensitivity and $64 \%$ specificity for detecting synovitis and $77 \%$ sensitivity and $97 \%$ specificity for detecting soft-tissue impingement. ${ }^{23}$

\section{Management}

\section{Conservative}

Physical therapy (especially lateral ankle stability protocols), shock-absorbing shoes, steroid injection, NSAIDs, use of a heel-lift orthosis to prevent dorsiflexion, and activity restriction have all been advocated and seem to be reasonable nonsurgical options for patients with AAI.

\section{Surgical management}

Surgical intervention is considered in patients with persistent symptoms. Surgical goals include removing the exostoses and débriding the soft tissue in the anterior ankle joint. Patients with tibial, talar, and/or fibular exostoses may be asymptomatic, and softtissue inflammation and impingement may contribute significantly to the pain. ${ }^{24} \mathrm{~A}$ complete diagnostic arthroscopy should be performed to ensure a thorough examination for intra-articular pathology. Hypertrophic synovium is often encountered, and a shaver is used to débride this material to enhance visualization. The anterior, medial, lateral, and posterior compartments are thoroughly débrided.
Osteochondral lesions are microfractured, nonviable cartilage flaps trimmed, and loose bodies removed. No studies describe how much bone should be removed from the anterior distal tibia or talar neck. We routinely use a $3.5-\mathrm{mm}$ arthroscopic burr to contour the anterior tibia until it is flush with the anterior border of the medial malleolus. ${ }^{25}$ Bossing or exostoses of the talar neck are débrided so that there is no tibial talar contact at maximal dorsiflexion. If the impinging bone and hypertrophic soft tissue cannot be adequately débrided using the standard anteromedial and anterolateral portals, accessory portals may be created to access the ankle joint. Extending either the lateral or the medial portal to create an open arthrotomy allows rapid and complete débridement of such pathology. In cases of lateral ankle instability, tibial, fibular, talar, and soft-tissue impingement can be easily removed through the standard extensile lateral arthrotomy, after which ligament reconstruction is performed. ${ }^{26}$

\section{Rehabilitation}

Despite the critical importance of postoperative treatment, no studies specifically address the rehabilitation protocol after removal of exostoses or soft-tissue impingement. ${ }^{27}$ Some surgeons allow weight bearing as tolerated following surgery, whereas others prefer nonweight bearing in a splint for 5 days followed by progressive weight bearing. Active and passive range of motion are begun soon after surgery, as are physical therapy (for strengthening) and proprioceptive exercises and aggressive range of motion and weight bearing.

\section{Outcomes}

In a series on arthroscopic débridement, of 12 patients (10 athletes) we had average $8^{\circ}$ improvement in ankle dorsiflexion, with 11 of 12 patients returning to sporting activities with a substantial improvement in pain at a mean of 3 months after surgery. Full athletic participation at a mean follow-up of 9 months after arthroscopic synovectomy with removal of exostoses and $92 \%$ were satisfied with their outcome. There were no complications in all series and there was no need for open arthrotomy of the joint for further debridement.

\section{Summary and conclusion}

AAI is a common condition characterized by chronic anterior ankle pain that is exacerbated by dorsiflexion. ${ }^{28}$ In the acute phase, pain is elicited following long periods of activity and relieved with rest. As the condition becomes chronic, patients report limited ankle motion and diminished exercise tolerance. They also report anterior ankle tenderness and pain with ankle dorsiflexion. Typically, radiographs demonstrate exostoses off the anterior distal tibia and dorsal talar neck. ${ }^{29}$ When nonsurgical measures are unsuccessful, surgical débridement of the offending soft tissues and exostoses has shown great success in returning patients to their previous levels of activity. Surgeons should carefully assess and simultaneously treat these patients for other foot and ankle pathology. ${ }^{30}$

\section{Acknowledgments}

None.

\section{Conflicts of interest}

None.

\section{References}

1. Baums MH, Kahl E, Schultz W, et al. Clinical outcome of the arthroscopic management of sports-related "anterior ankle pain": A prospective study. Knee Surg Sports Traumatol Arthrosc. 2006;14(5):482-486. 
2. Thomas MJ, Roddy E, Zhang W, et al. The population prevalence of foot and ankle pain in middle and old age: A systematic review. Pain. 2011;152(12):2870-2880.

3. Rasmussen S, Hjorth Jensen C. Arthroscopic treatment of impingement of the ankle reduces pain and enhances function. Scand $\mathrm{J}$ Med Sci Sports. 2002;12(2):69-72.

4. McMurray T. Footballer's ankle. J Bone Joint Surg Br. 1950;32(1):68-69.

5. Massada JL. Ankle overuse injuries in soccer players: Morphological adaptation of the talus in the anterior impingement. J Sports Med Phys Fitness. 1991;31(3):447-451.

6. Hawkins RB. Arthroscopic treatment of sports-related anterior osteophytes in the ankle. Foot Ankle. 1988;9(2):87-90.

7. Cannon LB, Hackney RG. Anterior tibiotalar impingement associated with chronic ankle instability. J Foot Ankle Surg. 2000;39(6):383-386.

8. Sommer C, Hintermann B, Nigg BM, et al. Influence of ankle ligaments on tibial rotation: An in vitro study. Foot Ankle Int. 1996;17(2):79-84.

9. Bassett FH, Gates HS, Billys JB, et al. Talar impingement by the anteroinferior tibiofibular ligament: A cause of chronic pain in the ankle after inversion sprain. J Bone Joint Surg Am. 1990;72(1):55-59.

10. Bonnel F, Toullec E, Mabit C, et al. Chronic ankle instability: Biomechanics and pathomechanics of ligaments injury and associated lesions. Orthop Traumatol Surg Res. 2010;96(4):424-432.

11. Ogilvie-Harris DJ, Mahomed N, Demaziere A. Anterior impingement of the ankle treated by arthroscopic removal of bony spurs. J Bone Joint Surg Br. 1993;75(3):437-440.

12. O'Donoghue DH. Impingement exostoses of the talus and tibia. $J$ Bone Joint Surg Am. 1957;39-A(4):835-852.

13. Hua Y, Chen S, Li Y, et al. Combination of modified Brostrom procedure with ankle arthroscopy for chronic ankle instability accompanied by intra-articular symptoms. Arthroscopy. 2010;26(4):524-528.

14. Tol JL, van Dijk CN. Etiology of the anterior ankle impingement syndrome: A descriptive anatomical study. Foot Ankle Int 2004;25(6):382-386.

15. Berberian WS, Hecht PJ, Wapner KL, et al. Morphology of tibiotalar osteophytes in anterior ankle impingement. Foot Ankle Int. 2001;22(4):313-317.

16. Stoller SM, Hekmat F, Kleiger B. A comparative study of the frequency of anterior impingement exostoses of the ankle in dancers and non dancers. Foot Ankle. 1984;4(4):201-203.
17. Bauer T, Breda R, Hardy P. Anterior ankle bony impingement with joint motion loss: The arthroscopic resection option. Orthop Traumatol Surg Res. 2010;96(4):462-468.

18. Moon JS, Lee K, Lee HS, et al. Cartilage lesions in anterior bony impingement of the ankle. Arthroscopy. 2010;26(7):984-989.

19. Branca A, Di Palma L, Bucca C, et al. Arthroscopic treatment of anterior ankle impingement. Foot Ankle Int. 1997;18(7):418-423.

20. O'Kane JW, Kadel N. Anterior impingement syndrome in dancers. Curr Rev Musculoskelet Med. 2008;1(1):12-16.

21. van Dijk CN, Wessel RN, Tol JL, et al. Oblique radiograph for the detection of bone spurs in anterior ankle impingement. Skeletal Radiol. 2002;31(4):214-221.

22. Nihal A, Rose DJ, Trepman E. Arthroscopic treatment of anterior ankle impingement syndrome in dancers. Foot Ankle Int. 2005;26(11):908-912.

23. Sanders TG, Rathur SK. Impingement syndromes of the ankle. Magn Reson Imaging Clin N Am. 2008;16(1):29-38.

24. Huh YM, Suh JS, Lee JW, et al. Synovitis and soft tissue impingement of the ankle: Assessment with enhanced three dimensional FSPGR MR imaging. J Magn Reson Imaging. 2004;19(1):108-116.

25. van Dijk CN, Tol JL, Verheyen CC. A prospective study of prognostic factors concerning the outcome of arthroscopic surgery for anterior ankle impingement. Am J Sports Med. 1997;25(6):737-745.

26. Lee J, Hamilton G, Ford L. Associated intra-articular ankle pathologies in patients with chronic lateral ankle instability: Arthroscopic findings at the time of lateral ankle reconstruction. Foot Ankle Spec. 2011;4(5):284-289.

27. Reynaert P, Gelen G, Geens G. Arthroscopic treatment of anterior impingement of the ankle. Acta Orthop Belg. 1994;60(4):384-388.

28. Ferkel RD, Chams RN. Chronic lateral instability: Arthroscopic findings and longterm results. Foot Ankle Int. 2007;28(1):24-31.

29. Haverstock BD. Anterior ankle abutment. Clin Podiatr Med Surg. 2001;18(3):457-465.

30. Sandmeier RH, Renstrom PA. Ankle arthroscopy. Scand J Med Sci Sports. 1995;5(2):64-70. 